\title{
Numerical Study on Influence of Hydrofoil Clearance Towards Total Drag Reduction on Winged Air Induction Pipe for Air Lubrication
}

\author{
Yanuar $^{1 *}$, Made S.G. Putra ${ }^{1}$, M Akbar $^{1}$, Muhammad Alief ${ }^{1}$, Fatimatuzzahra ${ }^{1}$ \\ ${ }^{1}$ Department of Mechanical Engineering, Faculty of Engineering, Universitas Indonesia, Kampus UI Depok, \\ Depok 16424, Indonesia
}

\begin{abstract}
A new device for air lubrication called the Winged Air Induction Pipe (WAIP) is studied in the present work. The device, which consists of an angled hydrofoil, uses the low-pressure region produced above the hydrofoil as a ship moves forward. The low pressure drives the atmospheric air into the water at certain velocities when the pressure is negative compared to atmospheric pressure. A computational fluid dynamics approach is presented in the study of the effect of the hydrofoil clearance of the Winged Air Induction Pipe on the drag reduction experienced by the plate to which the WAIP is attached. The well-known 'volume of fluid' model and k- $\omega$ SST (shear stress transport) turbulence closure model were used in the 2D numerical simulation in ANSYS Fluent. The numerical simulation was conducted with different hydrofoil clearance and angle of attack configurations, and the effects of these parameters on total drag force and drag reduction are reported. The reduction of drag force is found to increase by about $10 \%$ compared to the bare plate configuration.
\end{abstract}

Keywords: Air lubrication; Computational fluid dynamics; Drag reduction; Hydrofoil; Multiphase flow

\section{Introduction}

Methods of drag reduction using air lubrication are becoming a promising study area due to the increase in fuel efficiency produced as the result of reduced drag. The principle of the air lubrication method is to reduce the Reynolds shear stress in a turbulent boundary layer of the flow (Yanuar et al., 2012). The magnitude of such stress can be moderately changed by the dispersed phase for the dilute two-phase flow, but the distribution pattern remains unchanged (Muste et al., 2009). Kodama et al. (2000) obtained promising results using air lubrication in the form of microbubbles for drag reduction. It is well known that the presence of air in the turbulent boundary layer of the flow leads to drag reduction for two reasons: first, by lowering the average viscosity and density of the mixture flow, with the mixture of gas and liquid having a lower density and viscosity compared to the liquid itself; and second, by decreasing the magnitude of the Reynolds shear stress through the interaction of the air and liquid.

A numerical study has been made as an alternative to the experimental approach, as it requires less time, while still producing accurate results by first conducting validation of similar experimental results. Various numerical studies have been performed to calculate

*Corresponding author's email: yanuar@eng.ui.ac.id, Tel.: +62-21-7270032; fax: +62-21-7270033 doi: 10.14716/ijtech.v11i1.1870 
drag reduction using various air lubrication methods. Mohanarangam et al. (2009) studied the phenomenon of drag reduction by the injection of microbubbles into the turbulent boundary layer using an Eulerian-Eulerian two-fluid model. Pang et al. (2014) investigated microbubble drag reduction using the Euler-Lagrange two-way coupling method in order to understand the drag reduction mechanism from such bubbles. Shereena et al. (2014) conducted a numerical simulation using k- $\omega$ SST to calculate the drag reduction produced by an air jets on axisymmetric-underwater vehicles. Air lubrication requires an injection to disperse air into the water; this requires energy due to the relatively high pressure in the water, particularly at certain depths in a ship's bottom hull. Pressure from an air compressor is required in order to inject air into the water. However, the amount of energy required is large enough to cancel out the energy saved by the air lubrication. The injection of air into water at certain depths requires various sources of energy: first the adiabatic compression, the air generation in the water and mechanical losses at the air compressor (Kumagai et al., 2015). As a result, net power savings fall to as little as $5 \%$.

Kumagai et al. (2015) developed a new device called the Winged Air Induction Pipe (WAIP). This consists of an air pipe and angled hydrofoil with a lower pressure at the upper surface due to the higher magnitudes of velocity. Previously, numerous studies on the effect of the hydrofoil on the air-water interface have been made. Duncan (1981) conducted an experiment on the breaking waves produced by a towed hydrofoil at constant depth and velocity, finding that the drag associated with the breaking was proportional to the downslope component of the weight of the breaking region. The wake survey measurement also showed that the drag associated with breaking reached more than three times the maximum drag that could theoretically be obtained with non-breaking waves. Kumagai et al. (2011) found that the hydrofoil also produced negative pressure that pulled air into the water as the hydrofoil was positioned near the water surface.

In this work, the WAIP from the previous work of Kumagai et al. (2015) is studied. The device produces natural air injection without using an air compressor at critical velocity Uc, which is defined as:

$$
U_{C}=\sqrt{\frac{2 g H \alpha}{C_{P} \alpha-\left(\frac{L}{h_{b}}\right) C_{D} \sin \theta}}
$$

where $g$ is gravity acceleration, $H$ is the depth of the injection, $\alpha$ is the mean void fraction, $C_{P}$ is the pressure coefficient, and $L, h_{b}, C_{D}$ and $\theta$ are the hydrofoil chord length, the air-water mixture layer thickness, coefficient drag and the hydrofoil angle of attack, respectively. However, Shereena et al. (2014) found that in some cases hydrofoils develop problems regarding the clearance to the bottom plate where the is WAIP located.

Following the previous research of Kumagai et al. (2015), optimalization of the device is yet to be made. Therefore, it should be noted that the numerical simulation conducted in this work aims to analyze the influence of the hydrofoil clearance in the WAIP on the amount of drag reduction produced and the relationship between the angle of attack and clearance of the hydrofoil in the device.

\section{Methods}

To simulate two phase flow, the Volume of Fluid (VOF), as implemented in ANSYS Fluent, was used, which can be employed to model the separation of air and water above and below the ship. The water was implemented as the primary phase and air as the 
secondary. Surface tension modeling was also used to achieve a representation of the airwater contour.

\subsection{Governing Equations}

Basic three dimensional, transient, viscous, incompressible, and two-phase immiscible fluid flow were numerically solved by discretizing RANS equations:

$$
\begin{gathered}
\nabla \cdot \boldsymbol{U}=0 \\
\frac{\partial \rho \boldsymbol{U}}{\partial t}+\nabla \cdot\left(\rho \boldsymbol{U} \boldsymbol{U}^{\boldsymbol{T}}\right)=-\nabla p^{*}+\nabla \cdot(\mu \nabla \boldsymbol{U})+\nabla \cdot(\rho \tau)+\boldsymbol{S}
\end{gathered}
$$

where $\boldsymbol{U}=\left(u_{x}+u_{y}+u_{z}\right)$ is the velocity vector on three axes, $t$ is the time, $\nabla$ is a vector differential operator, $p^{*}$ is relative pressure, and $\rho$ and $\mu$ are fluid properties of the density and the dynamic viscosity respectively. $\tau$ is the Reynolds stress tensor for turbulence flow. Closure of the turbulence model for $\tau$ is $k$ - $\omega$ Shear Stress Transport (SST). The turbulence kinetic energy $k$ and specific dissipation rate $\omega$ are estimated from the boundary conditions of turbulence quantities of the level of turbulence intensity $I$ and length scale $l$. In two dimensional simulations, the $\boldsymbol{U}$ vector only consists of $u_{x}$ and $u_{y}$, with the value of $u_{z}$ being zero.

To simulate turbulent flow, the Shear-Stress Transport (SST) $k$ - $\omega$ was used to model the near wall region of the flow. This was based on a previous study that found this model to be well suited to simulating two phase flows (Mohanarangam et al., 2009). The $k-\omega S S T$ model is an effective blend of a robust and accurate formulation of the $k$ - $\omega$ in the near wall region and $k-\omega$ model in the far field (Shereena et al., 2014). The $k$ - $\omega$ SST model gives more realistic results in the prediction of void fractions that occur in the near wall region (Menter, 1994). Instead of using an empirical wall function to correlate the near wall and far field regions, $k$ - $\omega$ SST solved two turbulence scalars directly on the wall boundary (Mohanarangam et al., 2009).

\subsection{Computational Domain}

Two-dimensional simulation was used to obtain clear pressure and speed distribution around the hydrofoil and induction pipe. The analyzed object was simplified by dividing the model into four parts, as shown in Figure 1. Part A $(2,216 \mathrm{~mm}$ of length) is the bare plate, part $\mathrm{B}(80 \mathrm{~mm})$ is the WAIP, part $\mathrm{C}(2,600 \mathrm{~mm})$ is a bare flat plate that receives the effect of the WAIP, and part D (184 $\mathrm{mm})$ is the afterbody. The overall model surface is defined as a non-slip wall.
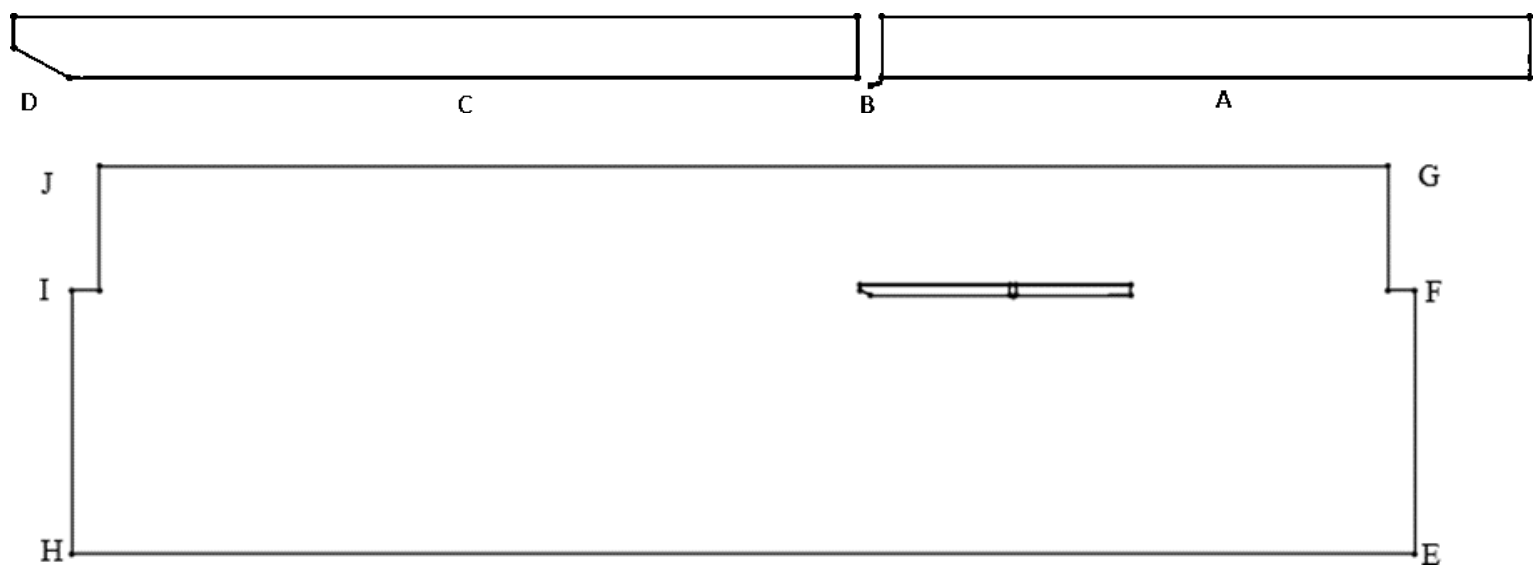

Figure 1 Computational Domain 
The computational domain used two different plane lengths to separate the air and water. In Figure 1, the segment EFIH is determined as the water boundary with 0 volume fraction of air, while segment FGJI is the air boundary with 1 volume fraction of air. The boundary conditions are defined as a non-slip wall, except: (a) segment EF is a $5.6 \mathrm{~m} / \mathrm{s}$ velocity inlet in the $-x$ direction with 0 volume fraction of air; and (b) segment $\mathrm{HI}$ is a pressure outlet.

\subsection{Grid and Discretization}

Before conducting the simulations, the sensitivity of the solution to the resolution of the grid was determined. The grid was refined with a ratio of 2 in every step of the refinement, from "coarse" to "finest" quantity, as shown in Table 1. The quality of the grid was determined by the non-dimensional wall parameter $\mathrm{y}^{+}$.

$$
y^{+}=\frac{\Delta y}{U} \sqrt{\frac{\tau_{w}}{\rho}}
$$

where $\Delta y$ is the distance from the first node to the wall. $\mathrm{y}^{+}$plays an important role in determining the accuracy of the numerical simulation, alongside the grid density. The grid independency test showed that the $\mathrm{y}^{+}$value decreased as the grid became refined, and increased slightly at the "finest" quantity. Simulation were conducted for all mesh qualities, with the results shown in Table 1.

Table 1 Grid sensitivity

\begin{tabular}{lccc}
\hline \multicolumn{1}{c}{ Mesh } & Grid Quantity & $\mathrm{y}^{+}$ & Solution (N) \\
\hline Finest & $4 \times 10^{5}$ & 2.92 & 155.53 \\
Fine & $2 \times 10^{5}$ & 2.84 & 156.63 \\
Fair & $1 \times 10^{5}$ & 3.32 & 158.02 \\
Medium & $5 \times 10^{4}$ & 4.23 & 160.92 \\
Coarse & $2.5 \times 10^{4}$ & 5.02 & 164.34 \\
\hline
\end{tabular}

The solutions of the coarse to the finest grids were compared to the $y+$ value to check the validity of the grid. The results show that the solutions displayed a relatively similar trend to the $y+$ value. As from the table, the solution changed significantly from the coarse to fine grid, whereas a small increment occurred from the fine to the finest grid. Hence, the "fine" grid quantity was chosen for the simulation.

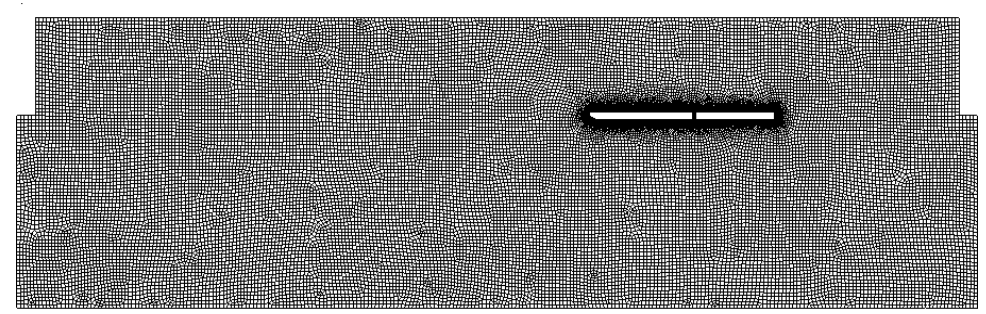

(a)

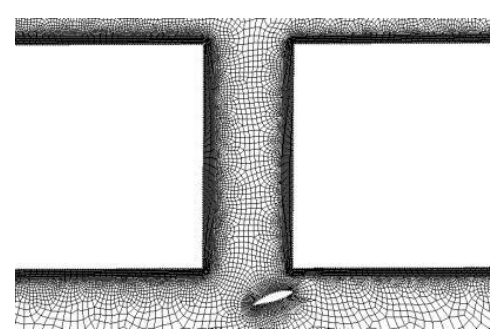

(b)

Figure 2 (a) Schematics diagram and mesh model (no. of nodes $=201775$; no. of elements = 196401); (b) Computational and enlarged view of mesh for part B (clearance $=20 \mathrm{~mm}$; angle of attack $=20^{\circ}$ ) 
The second order upwind scheme was used in all the calculations using pressure-based computation, and all the numerical simulations were conducted using a transient solution. The convergence criteria for the numerical parameters were all set at $10^{-3}$ for velocity, continuity, $\mathrm{k}$, and $\omega$ The time step used in the simulation was $0.001 \mathrm{~s}$, with the number of time steps 20, with each step having 20 iterations.

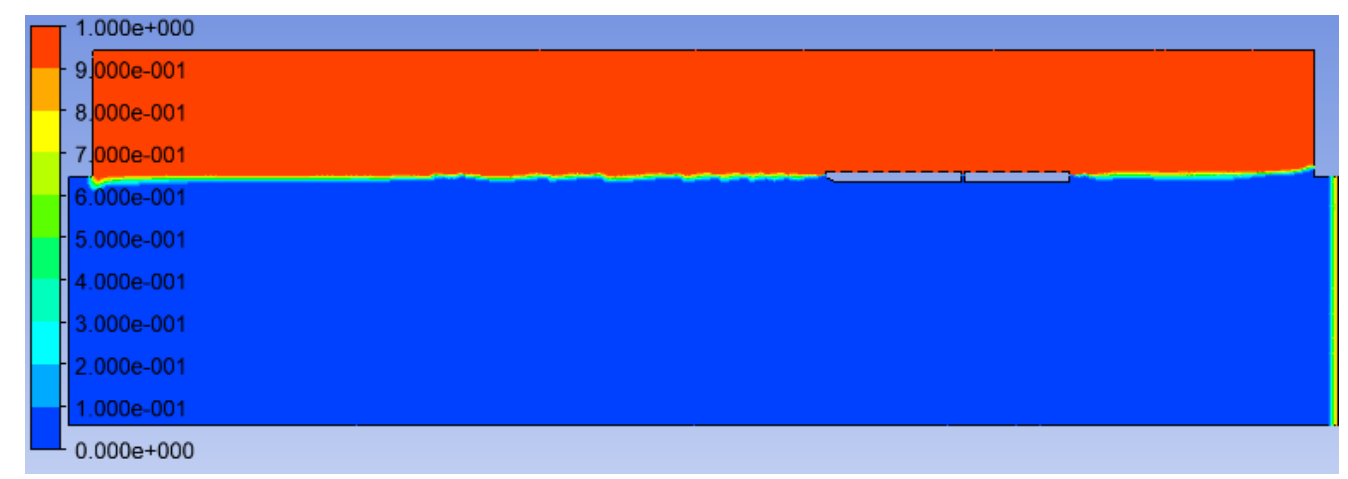

Figure 3 Two-dimensional representation of air-water interface in the computational domain

\subsection{Validation}

Kumagai et al. (2015) conducted an experimental study of the drag reduction measurement produced by the WAIP in part $\mathrm{C}$. Their results show the magnitude of drag experienced by part $C$ for each configuration of the WAIP. The bare plate configuration is also presented for the purpose of calculating drag reduction, where $\mathrm{Db}$ is the drag of part $\mathrm{C}$.

Table 2 Experimental data

\begin{tabular}{lccccc}
\hline \multicolumn{5}{c}{ Experimental Data with 15} & mm of Clearance (Kumagai et al., 2015) \\
\hline \multicolumn{1}{c}{ Type } & $\theta^{\circ}$ & $\begin{array}{c}\mathrm{v} \\
(\mathrm{m} / \mathrm{s})\end{array}$ & $\mathrm{Db}(\mathrm{N})$ & $\begin{array}{c}\Delta \mathrm{Db} \\
(\mathrm{N})\end{array}$ & $\begin{array}{c}\mathrm{DR} \\
(\%)\end{array}$ \\
\hline Bare Plate & Bare & 5.6 & 181.49 & - & - \\
WAIP & 12 & 5.6 & 167.75 & -13.73 & 7.57 \\
WAIP & 16 & 5.6 & 166.77 & -14.72 & 8.11 \\
WAIP & 20 & 5.6 & 162.85 & -18.64 & 10.27 \\
\hline
\end{tabular}

Numerical simulation of the same geometry was adopted and similar simulations were performed for validation purposes.

Table 3 Numerical data

\begin{tabular}{lcccccc}
\hline \multicolumn{7}{c}{ Numerical Data } \\
\hline Type & $\theta^{\circ}$ & $\begin{array}{c}\mathrm{v} \\
(\mathrm{m} / \mathrm{s})\end{array}$ & $\mathrm{Db}(\mathrm{N})$ & $\Delta \mathrm{Db}(\mathrm{N})$ & $\Delta \mathrm{R}(\%)$ & Error \\
\hline Bare Plate & Bare & 5.6 & 173.48 & - & - & $4.41 \%$ \\
WAIP & 12 & 5.6 & 155.24 & -18.24 & 10.51 & $7.46 \%$ \\
WAIP & 16 & 5.6 & 156.63 & -16.86 & 9.72 & $6.08 \%$ \\
WAIP & 20 & 5.6 & 177.53 & 4.05 & -2.33 & $9.02 \%$ \\
\hline \multicolumn{1}{c}{ Error } & & & & & $6.74 \%$ \\
\hline
\end{tabular}


Since some vital data such as plate draught and the length of the afterbody were not reported in the Kumagai et al.'s (2015) paper, the validation is somewhat approximate and shows an error of $6.74 \%$. From the results obtained, it is concluded that the Volume of Fluid model and $k$ - $\omega$ SST turbulence model are the appropriate computational models for this particular case.

\section{Results and Discussion}

The simulation was conducted without performing air injection from the hull pipe in the WAIP device to prove that the critical velocity in Equation 1 could produce the phenomenon of air entrainment due to the negative pressure produced by the hydrofoil. The critical velocity of $5.6 \mathrm{~m} / \mathrm{s}$ is used in the simulation, in line with a previous experiment (Kumagai et al., 2010). The fluid flow which occurs on the downstream side of the hydrofoil is difficult to map as a certain function. Hydrofoils always have the tendency to experience a larger value of drag as the angle of attack increases (Ockfen and Matveev, 2009). A large number of variations in the angle of attack are needed to precisely establish the exact plot of the phenomenon. In a previous study, Kumagai et al. (2011) also stated that the fundamental flow physics concerning this facility has yet to be clarified because of the extremely complicated phenomena involved.

A contour plot of the dynamic pressure around part B is shown in Figure 4a. As can be seen, the pressure becomes lower in the hull pipe above the hydrofoil. This phenomenon is referred to as negative pressure, when the pressure is lower compared to that of its surroundings. Negative pressure is produced as the fluid moves towards the model. The hydrofoil produces lower pressure on its upper surface due to the higher velocity caused by the longer curvature. However, in Figure $4 b$, without the foil, we can see that there are no or little negative pressure created by the device.

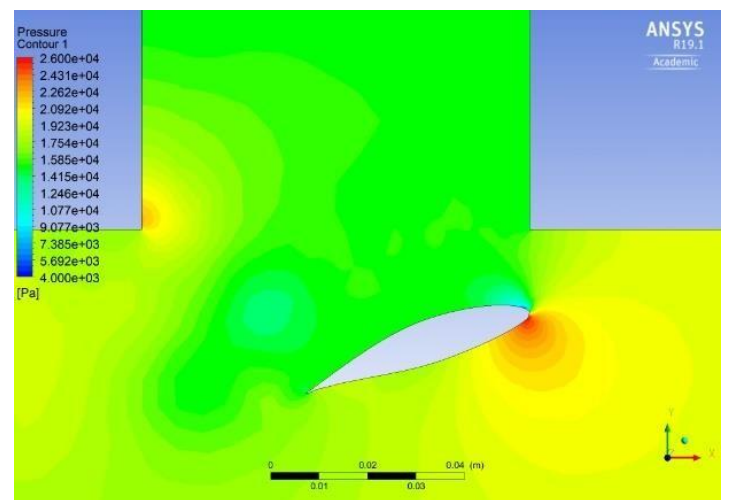

(a)

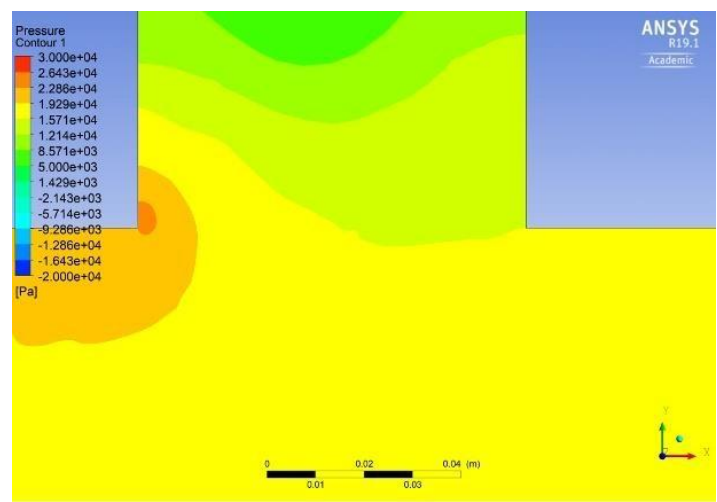

(b)

Figure 4 (a) Contour of dynamic pressure on part B; (b) pressure contour without foil

Table 4 shows the magnitude of drag reduction experienced by part $\mathrm{C}$ for each configuration of the model, where $D_{o}$, is the hydrofoil total drag. 
Table 4 Numerical results of the influence of hydrofoil clearance on the drag reduction of part C

\begin{tabular}{cccrccccc}
\hline Clearance $(\mathrm{mm})$ & $\theta^{\circ}$ & $\begin{array}{c}\mathrm{U} \\
\mathrm{(m} / \mathrm{s})\end{array}$ & \multicolumn{1}{c}{ Do } & \multicolumn{1}{c}{$\mathrm{Db}$} & $\mathrm{Dt}$ & \multicolumn{1}{c}{$\Delta$ Do } & \multicolumn{1}{c}{$\Delta \mathrm{Db}$} & DR (\%) \\
\hline \multirow{2}{*}{10} & bare & 5.60 & 174.76 & 173.48 & 348.24 & - & - & - \\
& 12 & 5.60 & 85.79 & 158.41 & 244.20 & -88.98 & -15.07 & 8.69 \\
& 16 & 5.60 & 127.41 & 159.42 & 286.82 & -47.35 & -14.06 & 8.11 \\
& 20 & 5.60 & 153.49 & 158.04 & 311.54 & -21.27 & -15.44 & 8.90 \\
\hline \multirow{2}{*}{15} & 12 & 5.60 & 76.15 & 155.24 & 231.39 & -98.62 & -18.24 & 10.51 \\
& 16 & 5.60 & 122.18 & 156.63 & 278.80 & -52.58 & -16.86 & 9.72 \\
& 20 & 5.60 & 465.42 & 177.53 & 642.95 & 290.66 & 4.05 & -2.33 \\
\hline \multirow{2}{*}{20} & 12 & 5.60 & 132.73 & 171.61 & 304.34 & -42.03 & -1.88 & 1.08 \\
& 16 & 5.60 & 221.34 & 170.40 & 391.74 & 46.58 & -3.08 & 1.78 \\
\hline
\end{tabular}

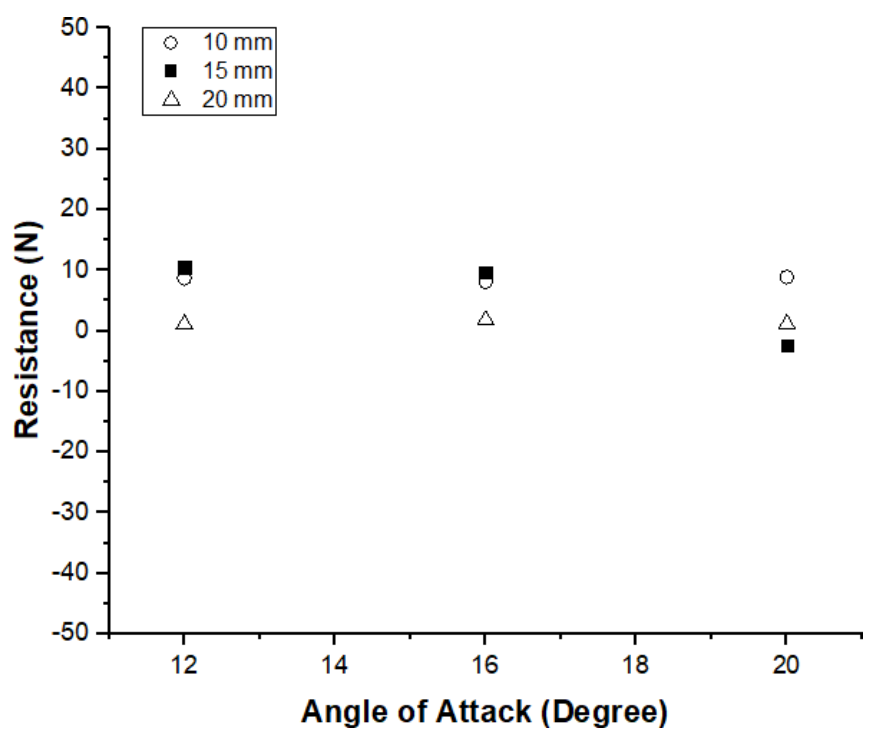

Figure 5 Drag reduction of part C

Table 4 shows the value of the drag reduction for each configuration of the model. On the clearance of $10 \mathrm{~mm} 8-9 \%$ of drag reduction is obtained. However, on the clearance of $15 \mathrm{~mm}$, there is an added drag of $2 \%$ on angle of attack of $20^{\circ}$. In this case the data is referred to as outliner or data that is outside the trend line (Mittink et al., 2001). Outliner is a common data in data distribution that do not follow the normal distribution. This can be caused by error from the numerical computation that has been performed where the mesh element relatively, is not evenly distributed. The distribution of mesh is depended on the element size of the mesh. Generally, as the element of the mesh gets smaller the more accurate the result gathered. The grid sensitivity test showed the finest grid does give a relatively small $\mathrm{y}+$ value. Since the $\mathrm{y}+$ correspond to determine the most appropriate simulation data, thus the "fine" quantity is chosen due to its small value. The error of the numerical data is less than 10 percent the result obtained is somewhat approximate regarding to the validation performed. In some modeling, to reach solution's grid independency, numerical value in the computational domain (the sizing parameter of mesh 
element) should be much smaller than corresponding local value of the model so that the numerical error could be minimized (Wang et al., 2012).

\section{Conclusions}

The paper has numerically investigated and optimized a WAIP device for possible use on the hull of the ship, with the clearance or distance from the hydrofoil's upper surface to the ship's hull the main study parameter. Clearance plays an important role in producing the appropriate amount of drag reduction. However, in some configurations the flow characteristic of the device produced more drag due to the depth variation of the hydrofoil.

The Computational Fluid Dynamics approach to estimate the drag reduction by air lubrication using the Winged Air Induction Pipe (WAIP) was taken and reasonably validated by the experimental work. By using nine configurations to achieve the effect of hydrofoil clearance on drag reduction, it is concluded that the desired magnitude of reduction can be achieved when the contributing parameters, namely the angle of attack and hydrofoil clearance, are optimally chosen. The numerical results were validated with published experimental results. Good agreement between these proves the accuracy of the numerical method employed in the calculation of the air-water interface and the results obtained.

The numerical results reveal that the optimum range is achieved by modification of the parameters using trial and error. The unique flow characteristic produced by the hydrofoil interacts with the Part $\mathrm{C}$ in different ways, depending on the clearance between the hydrofoil and the bottom plate of the model. The application of WAIP gives a level of net drag reduction of up to $10 \%$. In future work, 3D simulation is recommended to further study the effect of the size of the induction pipe at different positions and to explore the airwater interface phenomenon in its correlation to the drag reduction produced by the device.

\section{Acknowledgments}

The authors would like to thank Kementerian Riset, Teknologi dan Pendidikan Tinggi (KEMENRISTEKDIKTI). This work was supported by Indexed International Publication for Student Final Project NKB-1791/UN2.R3.1/HKP.05.00/2019.

\section{References}

Duncan, J., 1981. An Experimental Investigation of Breaking Waves Produced by a Towed Hydrofoil. In: Proceedings of the Royal Society A, Mathematical and Physical Science, Volume 377(1770), pp. 331-348

Kodama, Y., Kakugawa, A., Takahashi, T., Kawashima, H., 2000. Experimental Study on Microbubbles and their Applicability to Ships. Heat and Fluid Flow, Volume 21 pp. 582588

Kumagai, I., Kushida, T., Oyabu, K., Tasaka, Y., Murai, Y., 2011. Flow Behavior Around a Hydrofoil Close to a Free Surface. Visualization of Mechanical Processes, Volume 1(3), pp. 110-120.

Kumagai, I., Nakamura, N., Murai, Y., Tasaka, Y., Takeda, Y., 2010. A New Power-saving Device for Air Bubble Generation: Hydrofoil Air Pump for Ship Drag Reduction. In: International Conference on Ship Drag Reduction, SMOOTH-SHIPS, Istanbul, Turkey, pp. 93-102

Kumagai, I., Takahashi, Y., Murai, Y., 2015. Power-saving Device for air Bubble Generation using a Hydrofoil to reduce Ship Drag: Theory, Experiments, and Application to Ship. Ocean Engineering, Volume 95, pp. 183-194 
Menter, F., 1994. Zonal Two Equation Eddy Viscosity Turbulence Model for Engineering Applications. AIAA Journal, Volume 32, pp. 1598-1605

Mittink, S., Rachev, S.T., Samorodnitsky, G., 2001. The Distribution of Test Statistics for Outlier Detection in Heavy-tailed Samples. Mathematical and Computer Modeling, Volume 34(9-11), pp. 1171-1183

Mohanarangam, K., Cheung, S., Tu, J., Chen, L., 2009. Numerical Simulation of Microbubble Drag Reduction using Population Balance Model. Ocean Engineering, Volume 36, pp. 863-872

Muste, M., Yu, K., Fujita, I., Ettema, R., 2009. Two-phase Flow Insights into Open-channel Flows with Suspended Particles of Different Densities. Environ Fluid Mechanics, Volume 9(2) pp. 161-186

Ockfen, A.E., Matveev, K.I., 2009. Aerodynamics Characteristic of NACA 4412 Airfoil Section with Flap in Extreme Ground Effect. International Journal of Naval Architecture and Ocean Engineering, Volume 1(1) pp. 1-12

Pang, M., Wei, J., Yu, B., 2014. Numerical Study on Modulation of Microbubble on Turbulence Frictional Drag in a Horizontal Channel. Ocean Engineering, Volume 81, pp. 58-68

Shereena, S.G., Vengadesan, S., Idichandy, V.G., Bhattacharyya, S.K., 2014. CFD Study of Drag Reduction in Axissymetric Underwater Vehicles using Air Jets. Engineering Application of Computational Fluid Mechanics, Volume 7(2), pp. 193-209

Wang, H., Zhai, Z., 2012. Analyzing Grid Independency and Numerical Viscosity of Computational Fluid. Building and Environment, Volume 52, pp. 107-118

Yanuar, Gunawan, Sunaryo, Jamaluddin, A. 2012. Micro-bubble Drag Reduction on a High Speed Vessel Model. Journal of Marine Science and Application, Volume 11, pp. 301-304 